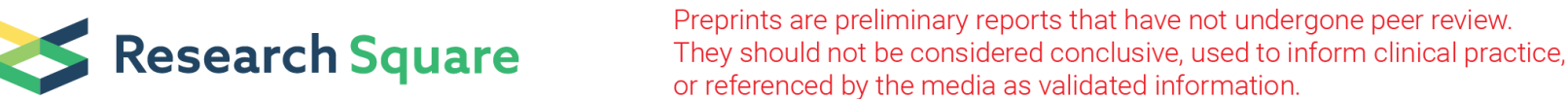

\section{Development and Validation a Prediction Model to Recurrence in Borderline Ovarian Tumor Patients with Fertility-Preserving Surgery}

Yunan He

Air Force Medical University https://orcid.org/0000-0002-5726-4539

Shunjie Hu

Sun Yat-sen University Zhongshan School of Medicine

Jiaojiao Zhong

Chinese Academy of Medical Sciences and Peking Union Medical College Hospital and Institute of

Dermatology: Chinese Academy of Medical Sciences \& Peking Union Medical College Hospital of Skin

Diseases and Institute of Dermatology

Hui Yang

Hunan University of Medicine

Nianchun Shan ( $\square$ shannc@csu.edu.cn )

Central South University

\section{Research}

Keywords: Borderline ovarian tumor, fertility-preserving surgery, recurrence, disease-free survival, prediction model

Posted Date: August 23rd, 2021

DOI: https://doi.org/10.21203/rs.3.rs-820789/v1

License: (c) (i) This work is licensed under a Creative Commons Attribution 4.0 International License. Read Full License 


\section{Abstract}

Background: To identify prognostic factors in patients with borderline ovarian tumor (BOT) and establish and validate a nomogram predicting recurrence in BOT patients treated with fertility-preserving surgery.

Methods: Patients with BOT who underwent surgery at two institutions between January 2000 and June 2017 were included and categorized into training and validation cohorts. Univariate log-rank test and Cox regression analysis were performed in the training cohort to identify prognostic factors, and a nomogram was developed to predict the recurrence rate. The model was validated by calculating the $\mathrm{C}$-index and drawing the calibration curve and receiver operating curve (ROC).

Results: In the multivariate Cox regression analysis, practice period, past history of benign ovarian disease, past history of benign breast disease, elevated CA125 levels, elevated CA199 levels, surgical methods, greater omentum resection, FIGO stage, postoperative pregnancy, and re-operation were independently associated with recurrence-free survival $(p<0.05)$. The aforementioned prognostic factors were used to develop a nomogram. The nomogram demonstrated a good ability to predict the risk of recurrence (training cohort C-index: 0.866 , validation cohort C-index: 0.920 ). The calibration curve suggested that the predicted recurrence-free survival was closely related to the actual recurrence. ROC analysis showed that the nomogram had a good discriminatory power with the area under curve between 0.776 and 0.956 .

Conclusions: The nomogram can predict the 1-, 3-, and 5-year recurrence-free survival of BOT patients undergoing fertility-preserving surgery. The predictive model can help guide surgical plans, postoperative monitoring, and prognostic evaluation of BOT patients.

\section{Introduction}

Borderline ovarian tumor (BOT) is defined as "an ovarian tumor exhibiting an atypical epithelial proliferation greater than that seen in the benign counterpart but without destructive stromal invasion" by the World Health Organization [13]. It shows histopathological characteristics and clinical behavior between benign ovarian tumors and invasive epithelial ovarian cancers [12] and represents $10 \%$ to $20 \%$ of ovarian neoplasms [11]. Pathologically, BOT has low malignant potential $[1,20]$ and clinically, BOT is characterized by presentation at an earlier stage and younger age at the time of diagnosis (10 years earlier than ovarian cancer). Previous studies have revealed that $78.9 \%$ of BOT patients were diagnosed at FIGO stage I and one-third of them were younger than 40 years $[18,19]$. Because of the pathological and clinical features, fertility-preserving surgery that preserves the uterus and at least a part of ovary can be considered for young patients who wish to retain their fertility and even for patients with advancedstage BOT $[3,12,23]$.

Although BOT has a favorable prognosis, up to $25 \%$ of patients relapse or die from the disease [14]. A higher recurrence rate with a shorter recurrence interval has been recorded in BOT patients treated with fertility-sparing surgery as compared to patients treated with radical surgery $[2,4$, 
6]. Therefore, it is important to establish a prediction model to distinguish the population at a higher risk of recurrence after fertility-preserving surgery. This is essential to correctly identify candidates suitable for fertility-preserving surgery.

Nomogram is an ideal model that can simplify statistical predictive models into a single numerical estimate of the probability of a clinical event [7]. To date, there have been no published studies using a nomogram as a recurrence prediction model for BOT patients who undergo fertility-preserving surgeries. Therefore, the objective of this study was to develop a nomogram that could predict the probability of recurrence in these patients.

\section{Methods}

\section{Study population}

The patients had signed a consent when they were hospitalized, agreed to use clinical data and specimens for scientific research, and promised that their private information would not be disclosed. This study was approved by the Medical Ethical Committee of Xiangya Hospital (No.202005065) and was registered in the Chinese Clinical Trial Registry (ChiCTR2000033446). Clinical records of patients with BOT (diagnosis confirmed histopathologically) between January 2000 and June 2017 were retrieved from the information systems of two institutions (The First Affiliated Hospital of Hunan University of Medicine and Xiangya Hospital, Central South University). Patients from Xiangya Hospital were included into the training cohort, and patients from The First Affiliated Hospital of Hunan University of Medicine were included into the validation cohort.

\section{Inclusion and exclusion criteria}

The inclusion criteria were defined as follows: (1) patients who underwent conservative surgical treatment, consisting of unilateral ovarian tumor resection, bilateral ovarian tumor resection, unilateral adnexal resection, or unilateral adnexal resection + contralateral ovarian tumor resection; (2) BOT patients with a definite postoperative pathological diagnosis; (3) patients with complete clinicopathological and follow-up data; and (4) patients who agreed to receive telephone interviews.

In addition, patients were excluded if they met the following exclusion criteria: (1) had missing clinical data and; (2) refused to follow up.

\section{Variables definition}

Disease-free survival (DFS) was defined as the time interval between the date of postoperative pathological diagnosis and the date of the last imaging examination without signs of recurrence, recorded in months. The practice period of the surgeon was recorded in years, and it 
was equal to the operation date of the patient from the time the surgeon first became an attending physician. Re-operation was defined as a comprehensive staging surgery performed after the first operation before tumor recurrence. The follow-up was terminated if there was any evidence of recurrence that was confirmed on imaging.

\section{Data collection}

The collected data included: (1) patient demographics like age, gravidity, breast disease history, and ovarian disease history; (2) clinical characteristics, including the FIGO stage (according to the International Federation of Gynecology and Obstetrics 2014 criteria [17]), tumor size (maximum diameter of tumor reported by CT or MRI or ultrasound), type of primary surgery, and postoperative pathological diagnosis; (3) follow-up content: details on treatment, relapse and postoperative fertility, including postoperative adjuvant therapy, time to relapse, natural or assisted pregnancy, delivery way, delivery date, and pregnancy complications; (5) practice period of the primary surgeon.

\section{Model Development}

Continuous variables, including age, practice period and tumor size were grouped into graded data at intervals of 10 years, 10 years, and $2 \mathrm{~cm}$, respectively. The patients in the training cohort were divided into the recurrence and non-recurrence groups. We used SPSS 26.0 (IBM Co., Armonk, NY, USA) to compare the difference between the recurrence group and the non-recurrence group, categorical variables were analyzed using the Chi-square test or Fisher's exact test, and the variables that showed statistically significant results in the univariate log-rank test were subsequently included in the multivariate Cox regression model. Differences were considered significant at a level of $\mathrm{P}<0.05$ and hazard ratios $(\mathrm{HR})$ were represented with their $95 \%$ confidence intervals $(95 \% \mathrm{CI})$. DFS curves were depicted using the Kaplan-Meier method and compared using the log-rank test.

After identifying significant factors related to DFS through multivariate analyses $(P<0.05)$, a nomogram for predicting the 1-, 3-, and 5-year DFS was constructed using the R v4.0 software (R Foundation for Statistical Computing, Vienna, Austria; http://www.r-project.org/) with the rms and survival package.

\section{Model validation}

Internal validation was performed using the Bootstrap method for repeated sampling 1000 times, and the calibration of the nomogram was evaluated by the Concordance index (C-index). The calibration curve was analyzed by plotting the nomogram predictions and the actual recurrence rates of BOT patients treated with fertility-preserving surgery. The range of C-index is $0.5-1$. Closer the $\mathrm{C}$-index is to 1 , better the discrimination between the model prediction results and the reality. The receiver operating characteristic 
curve (ROC) was drawn, and the precision (sensitivity and specificity) of the model was evaluated by calculating the area under curve (AUC). Closer the AUC value is to 1, better is the model's discrimination.

External validation was performed using the data of the validation cohort. Time and region of data collection in the validation cohort were different from those in the training cohort, but the inclusion and exclusion criteria were the same.

\section{Delivery methods and recurrence}

To investigate the relationship between the methods of delivery and recurrence, we used the Chi-square test of the four-grid to analyze if there was a significant difference in delivery methods between the recurrent and non-recurrent groups in the training cohort. A p-value of $<0.05$ was considered statistically significant.

\section{Results}

\section{Patient characteristics}

A total of 386 BOT patients received primary surgical treatment between January 2000 and June 2017 in The First Affiliated Hospital of Hunan University of Medicine and Xiangya Hospital. Among them, 123 patients were unreachable by phone, 14 patients refused follow up, and 2 patients were successfully followed up, but the clinical data were missing. Finally, 247 BOT patients were included in this retrospective study. Among them, 204 patients from Xiangya Hospital were included in the training cohort and 43 patients from The First Affiliated Hospital of Hunan University of Medicine were included in the validation cohort. The ratio of the two groups was approximately 4.7:1. The demographic, clinical characteristics, and experimental results of the training and the validation cohorts were similar (Table 1). 
Table 1

Specimen demographic, clinical characteristics

\begin{tabular}{|c|c|c|c|}
\hline & $\begin{array}{l}\text { Train Cohort } \\
(\mathrm{N}=204) \\
\mathrm{n}(\%)\end{array}$ & $\begin{array}{l}\text { Validation Cohort } \\
(\mathrm{N}=43) ; \\
\mathrm{n}(\%)\end{array}$ & $P$ \\
\hline Age (years, mean $\pm S D$ ) & $32 \pm 9.192$ & $27 \pm 2.121$ & 0.226 \\
\hline median of follow-up period (months) & 55 & 61 & 0.198 \\
\hline Preoperative Delivery History & $93(45.59 \%)$ & $23(53.49 \%)$ & $<0.001$ \\
\hline Recurrence & $62(30.39 \%)$ & $13(30.23 \%)$ & 0.983 \\
\hline FIGO stage & & & 0.421 \\
\hline la & $108(52.94 \%)$ & $17(39.53 \%)$ & \\
\hline $\mathrm{lb}$ & $56(27.45 \%)$ & $14(32.56 \%)$ & \\
\hline Ic & $32(15.69 \%)$ & $8(18.60 \%)$ & \\
\hline II & $4(1.96 \%)$ & $2(4.65 \%)$ & \\
\hline III & $4(1.96 \%)$ & $2(4.65 \%)$ & \\
\hline Pathological Pattern & & & $<0.001$ \\
\hline serous & $102(50.00 \%)$ & $13(30.23 \%)$ & \\
\hline mucinous & $75(36.76 \%)$ & $24(55.81 \%)$ & \\
\hline seromucinous & $21(10.29 \%)$ & $0(0.00 \%)$ & \\
\hline endometrioid & $0(0.00 \%)$ & $1(2.33 \%)$ & \\
\hline borderline Brenner tumor & $0(0.00 \%)$ & $1(2.33 \%)$ & \\
\hline others & $6(2.94 \%)$ & $4(9.30 \%)$ & \\
\hline Surgical Methods & & & 0.785 \\
\hline UOTR & $34(16.67 \%)$ & $8(18.60 \%)$ & \\
\hline BOTR & 39 (19.11\%) & $8(18.60 \%)$ & \\
\hline UAR & $86(42.16 \%)$ & $15(34.88 \%)$ & \\
\hline UAR+COTR & $45(22.06 \%)$ & $12(27.91 \%)$ & \\
\hline Surgical Type & & & 0.064 \\
\hline Laparoscope & $93(45.59 \%)$ & $13(30.23 \%)$ & \\
\hline \multirow[t]{2}{*}{ Laparotomy } & $111(54.41 \%)$ & $30(69.74 \%)$ & \\
\hline & Page $6 / 18$ & & \\
\hline
\end{tabular}




\begin{tabular}{llll}
\hline CA125 Elevated & $49(24.02 \%)$ & $12(27.91 \%)$ & 0.591 \\
\hline CA199 Elevated & $18(8.82 \%)$ & $4(9.30 \%)$ & 0.920 \\
\hline CEA Elevated & $5(2.45 \%)$ & $0(0.00 \%)$ & 0.300 \\
\hline Pelvic Lymph Node Resection & $1(0.49 \%)$ & $9(20.93 \%)$ & $<0.001$ \\
\hline Appendix Abnormal Appearance & $0(0.00 \%)$ & $0(0.00 \%)$ & 0.563 \\
\hline Greater Omentum Resection & $62(30.39 \%)$ & $15(34.88 \%)$ & 0.376 \\
\hline Lesion Region & & & \\
\hline \multicolumn{1}{c}{ Unilateral } & $124(60.78 \%)$ & $23(53.49 \%)$ & $<0.001$ \\
\hline \multicolumn{1}{c}{ Bilateral } & $80(39.22 \%)$ & $20(46.51 \%)$ & 0.403 \\
\hline Hormone Drugs & $5(2.45 \%)$ & $7(16.28 \%)$ & 0.434 \\
\hline Postoperative Chemoradiotherapy & $28(13.73 \%)$ & $6(13.95 \%)$ & 0.006 \\
\hline Postoperative Pregnancy & $50(24.51 \%)$ & $13(30.23 \%)$ & 0.759 \\
\hline Past History of Breast Begin Disease & $27(13.24 \%)$ & $13(30.23 \%)$ & 0.225 \\
\hline $\begin{array}{l}\text { Past History of Ovarian Begin } \\
\text { Disease }\end{array}$ & $52(25.49 \%)$ & $10(23.26 \%)$ & $12(27.90 \% \bigotimes$ \\
\hline Re-operation & $40(19.61 \%)$ & & \\
\hline
\end{tabular}

UOTR: unilateral ovarian tumor resection

BOTR: bilateral ovarian tumor resection

UAR: unilateral adnexa resection

COTR: contralateral ovarian tumor resection

\section{Predictive factors for recurrence in BOT patients treated with fertility-preserving surgery}

The factors predicting recurrence based on the univariate analysis can be seen in Table 2 . In the univariate log-rank test analysis, age, practice period, past history of benign ovarian disease, past history of benign breast disease, elevated CA125, elevated CA199, surgical methods, greater omentum resection, disease laterality, FIGO stage, pathological pattern, postoperative pregnancy, and re-operation were significant prognostic factors for DFS. In the multivariate COX regression analysis, the independent prognostic factors predicting the DFS comprised all the above, except age and disease laterality (Table $3)$. 
Kaplan-Meier survival curves demonstrated that lower practice period, past history of benign ovarian or breast disease, elevated CA125 or CA199 levels, complex surgical method without greater omentum resection, higher FIGO stage, postoperative pregnancy, and re-operation had worse DFS (Figure 1).

Table 2

Univariate log-rank test analysis

\begin{tabular}{lll}
\hline Variable & $\chi 2$ & P value \\
\hline Age & 64.030 & 0.009 \\
\hline Practice period & 101.047 & $<0.001$ \\
\hline Preoperative delivery history & 0.018 & 0.893 \\
\hline Past history of ovrian begin history & 21.925 & $<0.001$ \\
\hline Past history of breast begin history & 29.268 & $<0.001$ \\
\hline CA125 elevated & 30.899 & $<0.001$ \\
\hline CA199 elevated & 12.584 & $<0.001$ \\
\hline CEA elevated & 2.328 & 0.312 \\
\hline Tumor size & 6.692 & 0.669 \\
\hline Surgical methods & 21.133 & $<0.001$ \\
\hline Surgical type & 1.574 & 0.210 \\
\hline Pelvic lymph node resection & 0.984 & 0.321 \\
\hline Greater Omentum resection & 11.937 & 0.001 \\
\hline Unilater or bilateral lesion & 8.793 & 0.003 \\
\hline FIGO stage & 31.177 & $<0.001$ \\
\hline Pathological pattern & 8.976 & 0.030 \\
\hline Postoperative chemoradiotherapy & 0.042 & 0.838 \\
\hline Postoperative pregnancy & 18.328 & $<0.001$ \\
\hline Hormone drugs & 0.467 & 0.494 \\
\hline Re-operation & 18.917 & $<0.001$ \\
\hline Pvalye less than & & \\
\hline Pan & & \\
\hline
\end{tabular}

$P$ value less than 0.05 is indicated by bold. 
Table 3

Multivariate Cox proportional hazards regression analysis

\begin{tabular}{|c|c|c|c|c|c|}
\hline & B & SE & Waldx2 & $\begin{array}{l}\mathrm{P} \\
\text { value }\end{array}$ & $\mathrm{HR}(95 \% \mathrm{Cl})$ \\
\hline Age (10-19y) & & & 4.63 & 0.327 & \\
\hline $20-29 y$ & -0.896 & 0.819 & 1.197 & 0.274 & $\begin{array}{l}0.408 \\
(0.082,2.032)\end{array}$ \\
\hline $30-39 y$ & -0.118 & 0.849 & 0.019 & 0.889 & $\begin{array}{l}0.888 \\
(0.168,4.694)\end{array}$ \\
\hline $40-49 y$ & -0.6 & 0.924 & 0.421 & 0.517 & $\begin{array}{l}0.549 \\
(0.090,3.361)\end{array}$ \\
\hline $50-59 y$ & -8.269 & 769.852 & $<0.001$ & 0.991 & $<0.001$ \\
\hline Practice period (0-10y) & & & 12.119 & 0.007 & \\
\hline $10-20 y$ & -0.967 & 0.404 & 5.727 & 0.017 & $\begin{array}{l}0.380 \\
(0.172,0.839)\end{array}$ \\
\hline $20-29 y$ & -1.38 & 0.501 & 7.602 & 0.006 & $\begin{array}{l}0.251 \\
(0.094,0.671)\end{array}$ \\
\hline $30-39 y$ & 0.327 & 0.824 & 0.158 & 0.691 & $\begin{array}{l}1.387 \\
(0.276,6.977)\end{array}$ \\
\hline
\end{tabular}

Past history of ovrian begin history

(no)
yes
$0.823 \quad 0.34$
$5.863 \quad 0.015$
2.277

$(1.170,4.433)$

Past history of breast begin history (no)

$\begin{array}{llllll}\text { yes } & 1.08 & 0.334 & 10.444 & 0.001 & 2.946 \\ & & & & & \end{array}$

CA125 elevated (no)

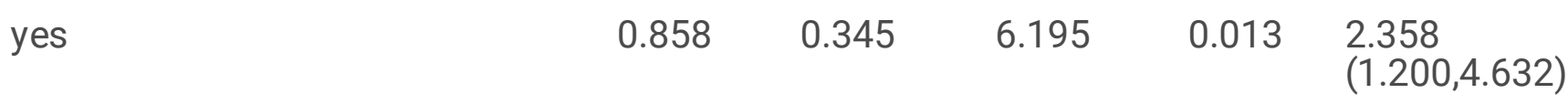

CA199 elevated (no)

$\begin{array}{llllll}\text { yes } & 1.134 & 0.4 & 8.018 & 0.005 & 3.108\end{array}$

$(1.418,6.814)$

Surgical methods (UOTR)

$12.125 \quad 0.007$

BOTR

$\begin{array}{llll}1.65 & 1.208 & 1.866 & 0.172\end{array}$

5.208

$(0.488,55.558)$ 


\begin{tabular}{|c|c|c|c|c|c|}
\hline UAR & -1.023 & 0.51 & 4.025 & 0.045 & $\begin{array}{l}0.359 \\
(0.132,0.977)\end{array}$ \\
\hline UAR+COTR & 2.275 & 1.189 & 3.662 & 0.056 & $\begin{array}{l}9.724 \\
(0.946,99.919)\end{array}$ \\
\hline
\end{tabular}

Greater Omentum resection (no)

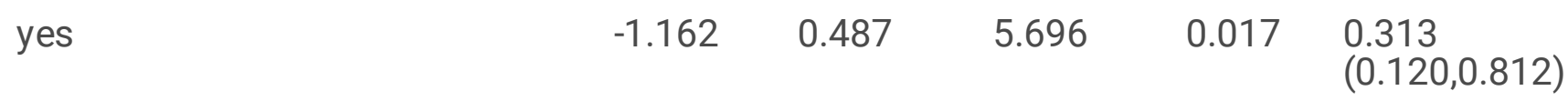

Unilater or bilateral lesion

(Unilateral)

$\begin{array}{llllll}\text { bilateral } & -1.071 & 1.158 & 0.855 & 0.355 & 0.343\end{array}$

$(0.035,3.316)$

FIGO stage (la)

$\mathrm{Ib}$

Ic

II

III

Pathological pattern (serousity)

mucinousness

serous mucinity

others

12.296

0.015

$-0.323 \quad 0.957$

0.114

0.736

0.724

$(0.111,4.726)$

$\begin{array}{llll}-0.914 & 0.873 \quad 1.098 & 0.295\end{array}$

$0.401(0.072$, 2.217)

$\begin{array}{lllll}2.112 & 0.746 & 8.019 & 0.005 & 8.261\end{array}$

$(1.916,35.626)$

$\begin{array}{lllll}0.163 & 1.03 & 0.025 & 0.874 & 1.177\end{array}$

$(0.156 .8 .857)$

$0.555 \quad 0.334$

$2.912 \quad 0.405$

$2.763 \quad 0.096$

$1.741(0.905$, 3.349)

$\begin{array}{lllll}-11.857 & 284.218 & 0.002 & 0.967 & <0.001\end{array}$

$\begin{array}{lllll}-0.166 & 1.094 & 0.023 & 0.88 & 0.847\end{array}$

$(0.099,7.233)$

Postoperative pregnancy (no)
yes
0.704
0.319
4.864
0.027
2.022

$(1.082,3.782)$

Re-operation (no)

$$
\text { yes }
$$

$$
-2.63
$$

1.099

5.722

0.017

0.072

$(0.008,0.622)$

B: regression coefficient

SE: standard error

UOTR: unilateral ovarian tumor resection

BOTR: bilateral ovarian tumor resection 
UAR: unilateral adnexa resection

COTR: contralateral ovarian tumor resection

$P$ value less than 0.05 is indicated by bold.

\section{Development and validation of the predictive nomogram}

All ten variables with statistical significance noted in the multivariable analysis for predicting the rate of recurrence were chosen to develop the nomogram (Figure 2). The C-index of the nomogram was 0.866 (95هCl: 0.824-0.908) in the training cohort. The model manifested a good degree of calibration. The AUC of the $1-, 3-$, and 5 -year DFS were $0.776,0.895$, and 0.940 , respectively, in the training cohort (Figure 3 ).

The performance of the nomogram was also evaluated in the validation cohort, with a C-index of 0.920 (95खCl: 0.824-1.000), indicating that the model had a good discrimination. In addition, the AUC of the 1-, 3 -, and 5-year DFS were $0.952,0.954$, and 0.956 , respectively, indicating good predictive ability of the model (Figure 4).

\section{Delivery methods and recurrence}

In the training cohort, there were 10 natural births and 20 cesarean sections in the recurrence group, and 16 natural births and 4 cesarean sections in the non-recurrence group. After Chi-square test, it was found that patients delivering with cesarean section post the fertility-preserving surgery were more likely to have tumor recurrence $(P=0.003)$.

\section{Discussion}

One of the clinical characteristics of BOT is that it tends to occur in women of childbearing age, making fertility-preserving surgery widely used in BOT patients, even in patients with advanced-stage disease [3, 16 ,23]. However, several studies have reported that fertility-preserving surgery is associated with an increased risk of recurrence $[4,22]$. Therefore, it is imperative to predict the probability of recurrence in these patients undergoing fertility-preserving surgery. In this study, we developed a nomogram that was capable of predicting tumor recurrence probability in BOT patients who were treated with fertilitypreserving surgery. All the predictive factors used in the model are closely related to clinical practice and are easy to obtain and use; thereby making this model potentially popular in routine clinical practice.

In the nomogram, re-operation is the most important protective factor; hence, we recommend that BOT patients with proven high-risk factors for recurrence should undergo comprehensive staging surgery after successful postoperative delivery. Patients who underwent unilateral ovarian tumor resection were most 
likely to experience a relapse, while patients with unilateral adnexal resection were the least likely. It is, therefore, suggested that unilateral adnexal resection should be performed in patients with fertility preservation needs if the contralateral ovary function is normal. We also found that unilateral or bilateral lesions were not associated with recurrence. Peritoneal implants have prognostic significance, and the most common sites of implants include the omentum and peritoneal surfaces; therefore, omentectomy was recommended in the comprehensive surgical staging [5]. However, another study, including 539 patients with BOT from 14 institutions concluded that comprehensive surgical staging was not necessary for borderline ovarian tumors [10]. We also believe that a comprehensive surgical staging with at least greater omentum resection may be beneficial for BOT patients. Unfortunately, data on cytologic washings and peritoneal biopsies were lacking and not included in this study, but greater omentum resection was shown to be a protective factor. Based on the study findings, it is recommended that the omentum be removed at the same time when fertility-preserving surgery is performed. However, further validation of this is required with randomized control or higher-level studies to prove the benefit in BOT patients.

This study also introduces several novel predictors of recurrence. The practice period of surgeon has a considerable effect on the recurrence of BOT. Longer is the practice period lower is the risk of recurrence. Past history of benign ovarian or breast tumor has also shown to increase the probability of BOT recurrence. The specific mechanism for this, however, is unclear, and we suggest a possible relationship to hormones.

Age has been considered to be a predictive factor for the recurrence of BOT in many studies, with some studies reporting that younger patients have a worse outcome [9], while others reported that older patients were far worse [21]. Unlike the previous studies, age was not found to be a predictor of recurrence in this study. This was possibly because the age of the patients undergoing fertility-preserving surgery was relatively lower, and the effect of age on tumor recurrence was not demonstrable. The primary surgery type performed by laparoscopy or by laparotomy is an independent factor for prediction of the risk of recurrence in our study, consistent with previous studies $[8,15]$.

Postoperative pregnancies were recorded in only a subset of patients, so the delivery methods were not included in the model and were analyzed using the Chi-square test. The result showed that cesarean section was significantly correlated with tumor recurrence. Although the sample size is limited, we should still pay attention to it. It is not recommended to relax the indications of cesarean section, to avoid tumor recurrence and metastasis in the pelvic cavity. Further studies are required on the relationship between the method of delivery and tumor recurrence.

The clinical prediction model of recurrence in BOT patients treated with fertility-preserving surgery conforms to the requirements of precision medicine and aims to achieve a better balance between the fertility requirements of the patients and the control of the disease. The AUC of our model is very high, indicating that this model can accurately predict the recurrence of BOT patients treated with fertilitypreserving surgery. However, some limitations need to be acknowledged. Due to limited data, the effects 
of immunohistochemistry and special pathological behaviors of tumor such as papillary structure and peritoneal implantation on the recurrence of BOT patients were not analyzed in this study. In addition, a small sample size and single-center characteristics may also affect the accuracy of this study. We hope that in the future, this nomogram can have an external multicenter validation.

\section{Conclusion}

The nomogram, designed in this article, is a good recurrence prediction model for BOT patients treated with fertility-preserving surgery. A high C-index and AUC indicate good discrimination and differentiation of the model. We recommend predicting the risk of recurrence for all BOT patients who want to preserve their fertility, and high-risk patients should undergo comprehensive staging surgery after successful postoperative delivery to avoid recurrence. Unilateral adnexal resection is the best surgical procedure for patients if the contralateral ovary function is normal.

\section{Declarations}

\section{Ethics approval and consent to participate}

This study was approved by the Medical Ethical Committee of Xiangya Hospital (No.202005065) and was registered in the Chinese Clinical Trial Registry (ChiCTR2000033446).

\section{Consent for publication}

All participating patients agree to use their medical personal information and follow-up, excluding private information.

\section{Availability of data and materials}

All data generated or analyzed during this study are included in this published article.

\section{competing interests}

The authors declare that they have no competing interests.

\section{Funding}

None.

\section{Authors' contributions}


YNH and NCS designed the study. YNH performed the experiments. JJZ and SJH analyzed the data. YNH, $\mathrm{SJH}, \mathrm{YH}$ wrote the manuscript. All authors reviewed and approved the final manuscript.

\section{Acknowledgements}

Thanks for all patients who provided clinical data for this article. We also would like to thank Editage (www.editage.cn) for English language editing.

\section{References}

1. (1971) Classification and staging of malignant tumours in the female pelvis. Acta Obstet Gynecol Scand 50(1):1-7. https://doi.org/10.3109/00016347109157278

2. du Bois A, Ewald-Riegler N, de Gregorio N, Reuss A, Mahner S, Fotopoulou C, et al. Hauptmann S (2013) Borderline tumours of the ovary: A cohort study of the Arbeitsgmeinschaft Gynakologische Onkologie (AGO) Study Group. Eur J Cancer 49(8):1905-1914. https://doi.org/10.1016/j.ejca.2013.01.035

3. du Bois A, Trillsch F, Mahner S, Heitz F, Harter P (2016) Management of borderline ovarian tumors. Ann Oncol 27 Suppl 1:i20-i22. https://doi.org/10.1093/annonc/mdw090

4. Fang C, Zhao L, Chen X, Yu A, Xia L, et al. Zhang P (2018) The impact of clinicopathologic and surgical factors on relapse and pregnancy in young patients $(</=40$ years old $)$ with borderline ovarian tumors. Bmc Cancer 18(1):1147. https://doi.org/10.1186/s12885-018-4932-2

5. Fotopoulou C, Schumacher G, Schefold JC, Denkert C, Lichtenegger W, et al. Sehouli J (2009) Systematic evaluation of the intraoperative tumor pattern in patients with borderline tumor of the ovary. Int J Gynecol Cancer 19(9):1550-1555. https://doi.org/10.1111/IGC.0b013e3181a84699

6. Guvenal T, Dursun P, Hasdemir PS, Hanhan M, Guven S, Yetimalar H, et al. Ayhan A (2013) Effect of surgical staging on 539 patients with borderline ovarian tumors: a Turkish Gynecologic Oncology Group study. Gynecol Oncol 131(3):546-550. https://doi.org/10.1016/j.ygyno.2013.08.038

7. Iasonos A, Schrag D, Raj GV, Panageas KS (2008) How to build and interpret a nomogram for cancer prognosis. J Clin Oncol 26(8):1364-1370. https://doi.org/10.1200/JC0.2007.12.9791

8. Jiao X, Hu J, Zhu L (2017) Prognostic Factors for Recurrence After Fertility-Preserving Surgery in Patients With Borderline Ovarian Tumors: A Systematic Review and Meta-analysis of Observational Studies. Int J Gynecol Cancer 27(9):1833-1841. https://doi.org/10.1097/IGC.0000000000001092

9. Karlsen N, Karlsen MA, Hogdall E, Nedergaard L, Christensen IJ, et al. Hogdall C (2016) Relapse and disease specific survival in 1143 Danish women diagnosed with borderline ovarian tumours (BOT). Gynecol Oncol 142(1):50-53. https://doi.org/10.1016/j.ygyno.2016.05.005

10. Kristensen GS, Schledermann D, Mogensen O, Jochumsen KM (2014) The value of random biopsies, omentectomy, and hysterectomy in operations for borderline ovarian tumors. Int J Gynecol Cancer 24(5):874-879. https://doi.org/10.1097/IGC.0000000000000140 
11. Lu Z, Li B, Gu C (2019) Outcomes of fertility-sparing surgery for stage II and III serous borderline ovarian tumors. J Int Med Res 47(10):4895-4903. https://doi.org/10.1177/0300060519865850

12. Matsuo K, Machida H, Mandelbaum RS, Grubbs BH, Roman LD, Sood AK, et al. Gershenson DM (2019) Mucinous borderline ovarian tumor versus invasive well-differentiated mucinous ovarian cancer: Difference in characteristics and outcomes. Gynecol Oncol 153(2):230-237. https://doi.org/10.1016/j.ygyno.2019.02.003

13. Mccluggage WG (2010) The pathology of and controversial aspects of ovarian borderline tumours. Curr Opin Oncol 22(5):462-472. https://doi.org/10.1097/CC0.0b013e32833b0dc1

14. Morice P, Uzan C, Fauvet R, Gouy S, Duvillard P, et al. Darai E (2012) Borderline ovarian tumour: pathological diagnostic dilemma and risk factors for invasive or lethal recurrence. Lancet Oncol 13(3):e103-e115. https://doi.org/10.1016/S1470-2045(11)70288-1

15. Odegaard E, Staff AC, Langebrekke A, Engh V, Onsrud M (2007) Surgery of borderline tumors of the ovary: retrospective comparison of short-term outcome after laparoscopy or laparotomy. Acta Obstet Gynecol Scand 86(5):620-626. https://doi.org/10.1080/00016340701286934

16. Park JY, Kim DY, Kim JH, Kim YM, Kim YT, et al. Nam JH (2009) Surgical management of borderline ovarian tumors: The role of fertility-sparing surgery. Gynecol Oncol 113(1):75-82. https://doi.org/10.1016/j.ygyno.2008.12.034

17. Prat J (2015) FIGO's staging classification for cancer of the ovary, fallopian tube, and peritoneum: abridged republication. J Gynecol Oncol 26(2):87-89. https://doi.org/10.3802/jgo.2015.26.2.87

18. Sherman ME, Berman J, Birrer MJ, Cho KR, Ellenson LH, Gorstein F, et al. Seidman JD (2004) Current challenges and opportunities for research on borderline ovarian tumors. Hum Pathol 35(8):961-970. https://doi.org/10.1016/j.humpath.2004.03.007

19. Sherman ME, Mink PJ, Curtis R, Cote TR, Brooks S, Hartge P, et al. Devesa S (2004) Survival among women with borderline ovarian tumors and ovarian carcinoma: a population-based analysis. CancerAm Cancer Soc 100(5):1045-1052. https://doi.org/10.1002/cncr.20080

20. Silverberg SG, Bell DA, Kurman RJ, Seidman JD, Prat J, Ronnett BM, et al. Young RH (2004) Borderline ovarian tumors: key points and workshop summary. Hum Pathol 35(8):910-917. https://doi.org/10.1016/j.humpath.2004.03.003

21. Song T, Lee YY, Choi CH, Kim TJ, Lee JW, Bae DS, et al. Kim BG (2014) Risk factors for progression to invasive carcinoma in patients with borderline ovarian tumors. Int J Gynecol Cancer 24(7):12061214. https://doi.org/10.1097/IGC.0000000000000216

22. Sun H, Chen X, Zhu T, Liu N, Yu A, et al. Wang S (2018) Age-dependent difference in impact of fertility preserving surgery on disease-specific survival in women with stage I borderline ovarian tumors. $J$ Ovarian Res 11(1):54. https://doi.org/10.1186/s13048-018-0423-y

23. Uzan C, Nikpayam M, Ribassin-Majed L, Gouy S, Bendifallah S, Cortez A, et al. Morice P (2014) Influence of histological subtypes on the risk of an invasive recurrence in a large series of stage I borderline ovarian tumor including 191 conservative treatments. Ann Oncol 25(7):1312-1319. https://doi.org/10.1093/annonc/mdu139

Page 15/18 
Figures
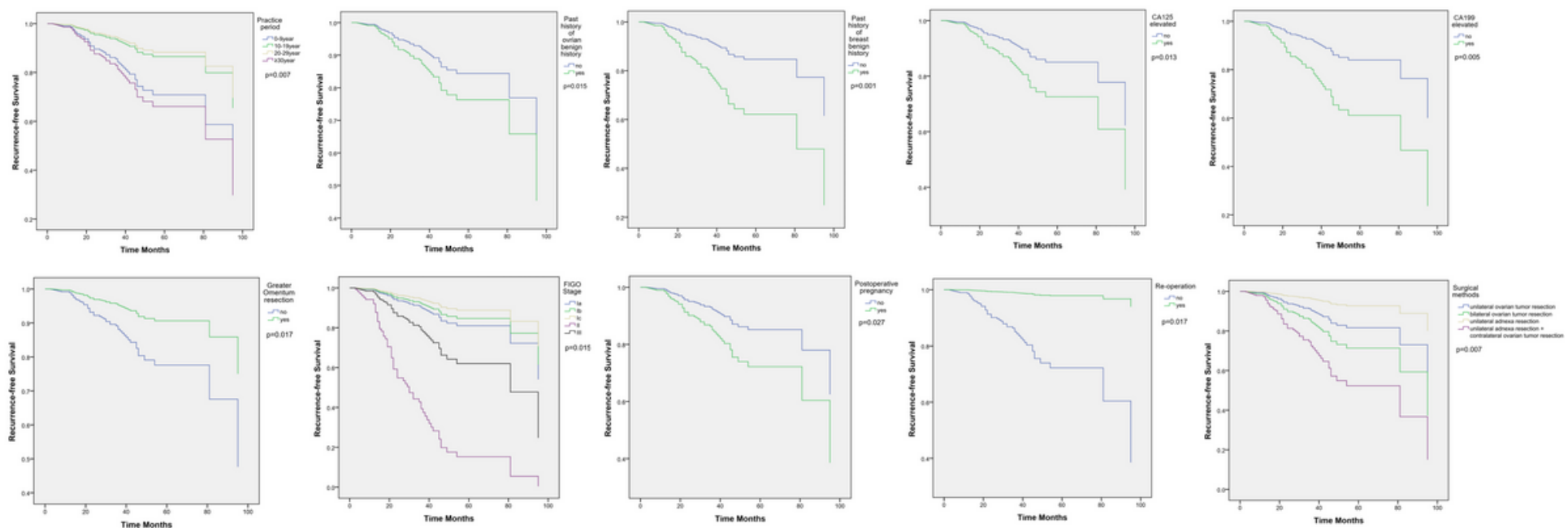

Figure 1

Kaplan-Meier disease-free survival curve of variable in training cohort.

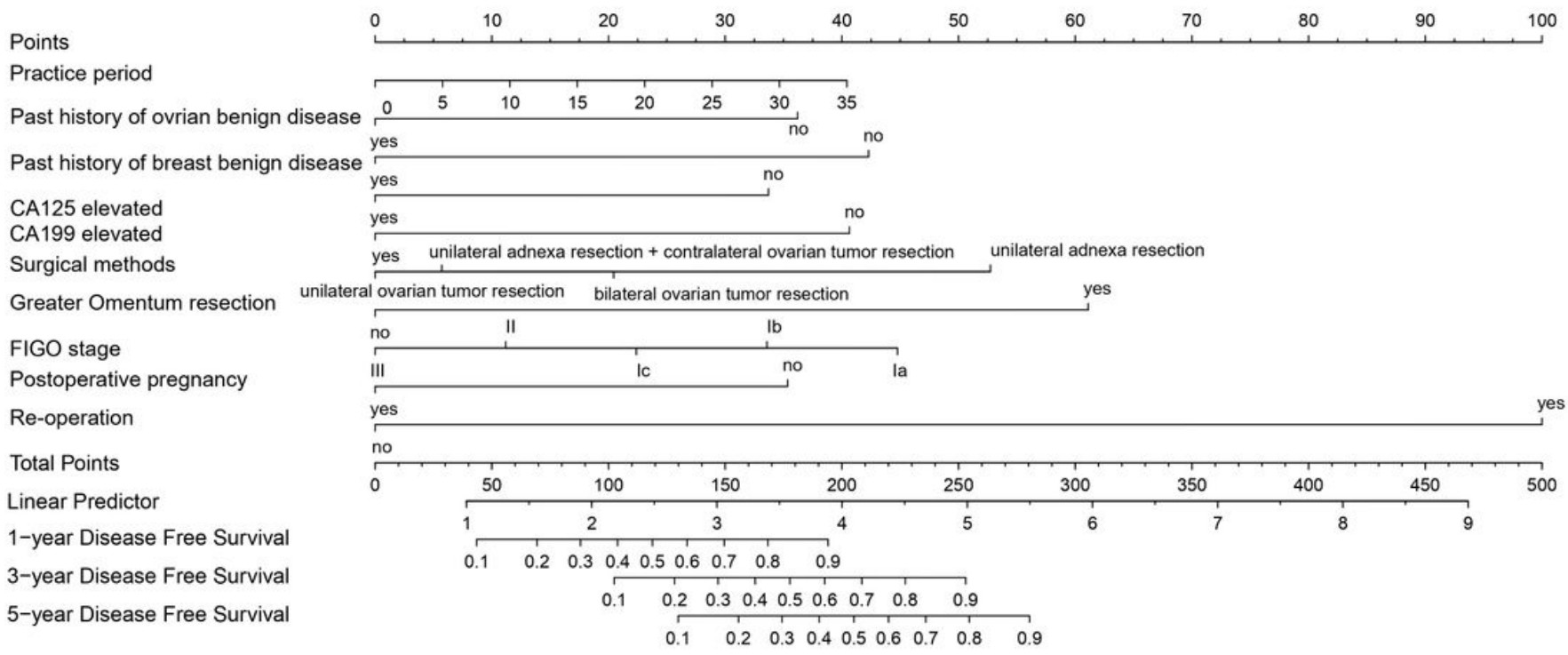

\section{Figure 2}

BOT patients treated with fertility-preserving surgery with nomogram including 10 readily available clinical characteristics to predict 1-, 3-, 5-year disease-free survival 
A

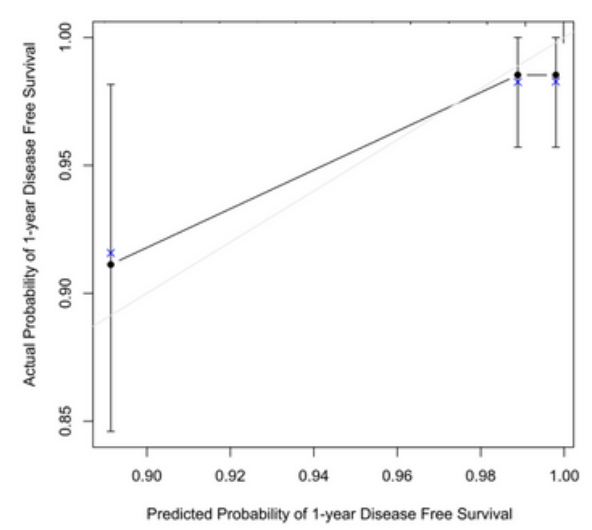

Year=1

B

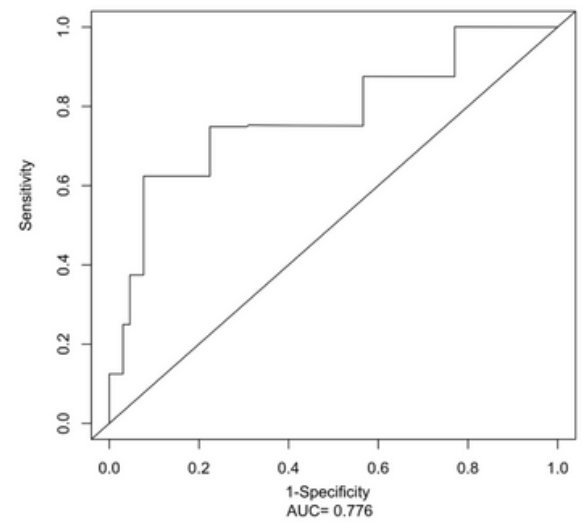

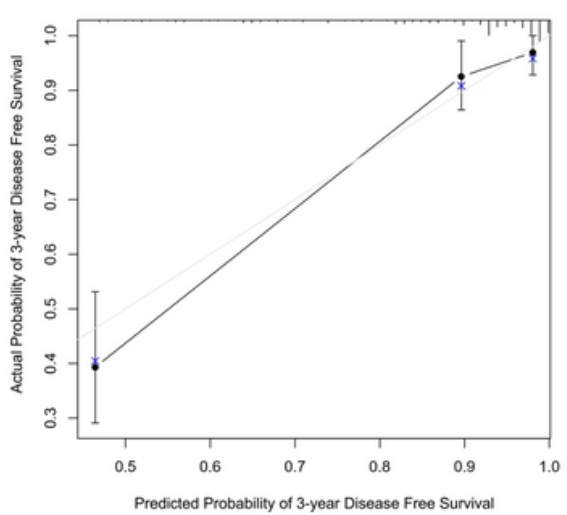

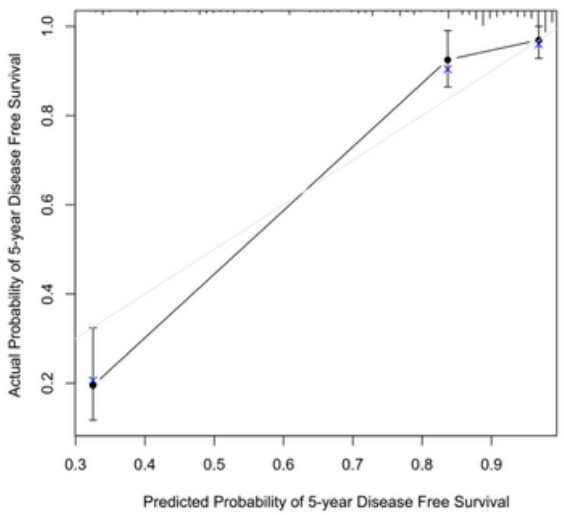

Year=3
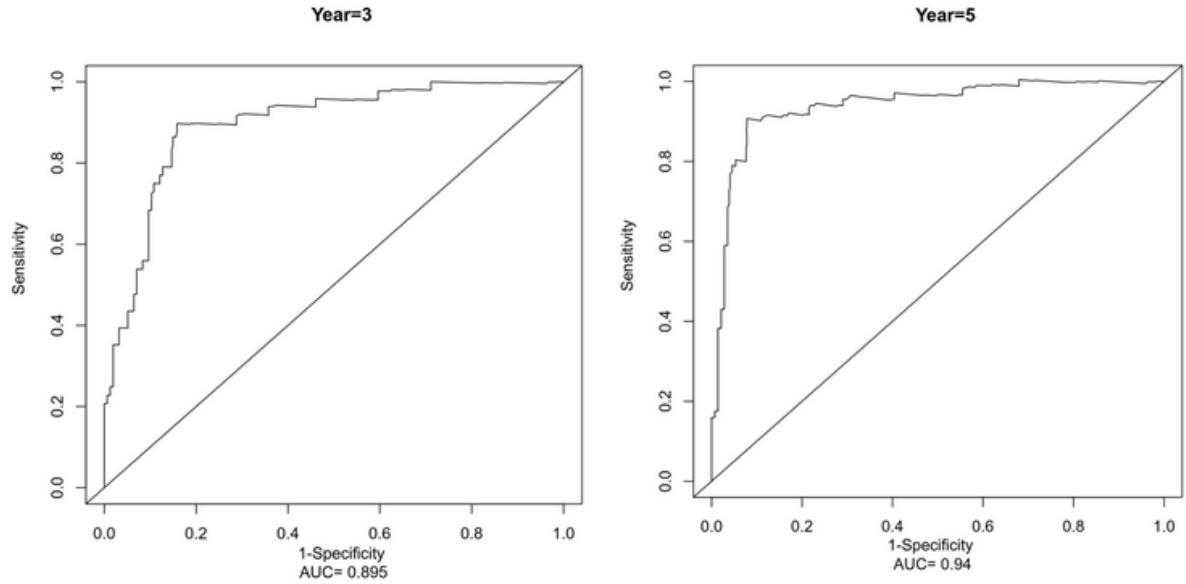

\section{Figure 3}

A. Calibration plot for the nomogram in the training cohort. The black line represents the actual DFS probability, whereas the gray line represents the DFS probability predicted using the nomogram. The two lines closely overlap, suggesting that the nomogram accurately estimated the recurrence probability of our patient cohort. B. The AUC of 1-, 3-, 5-year DFS in the training cohort. 
A

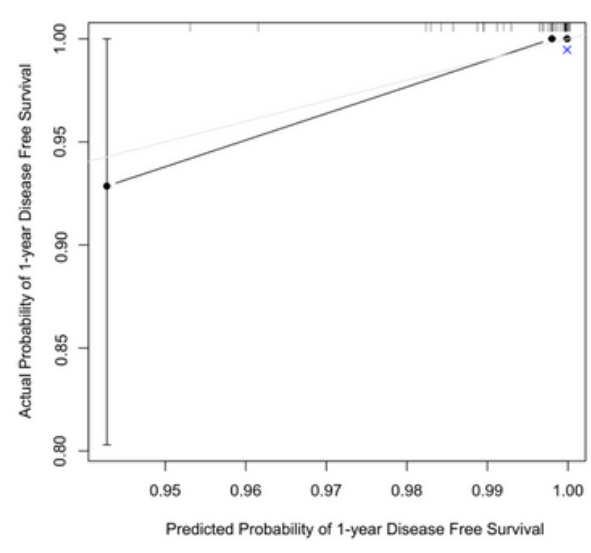

B

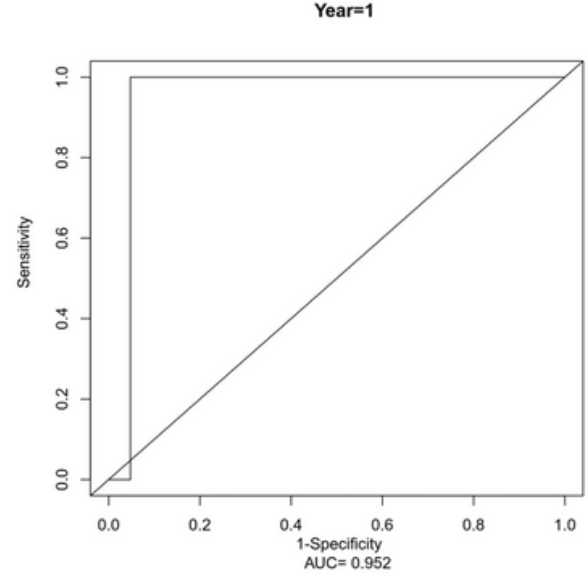

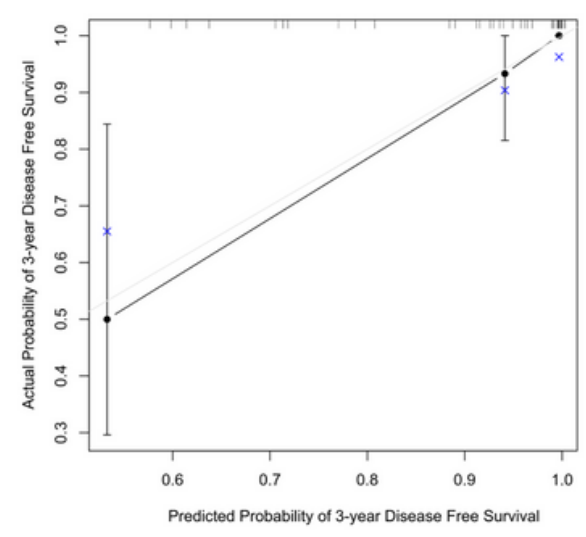

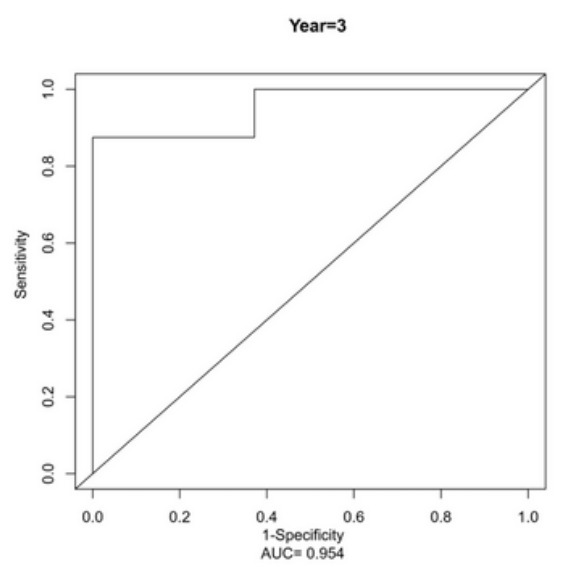

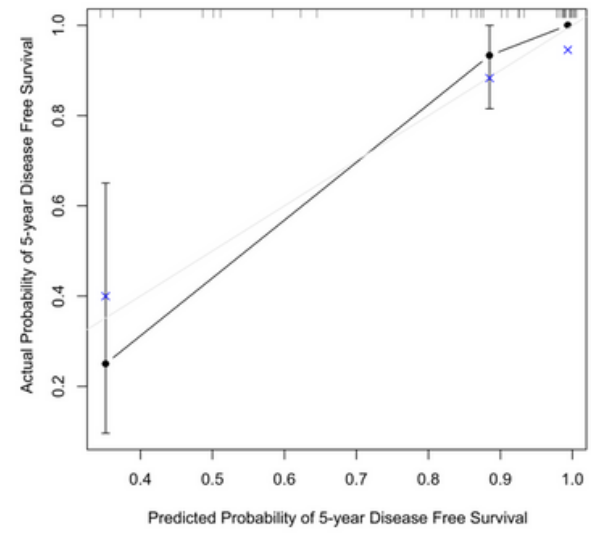

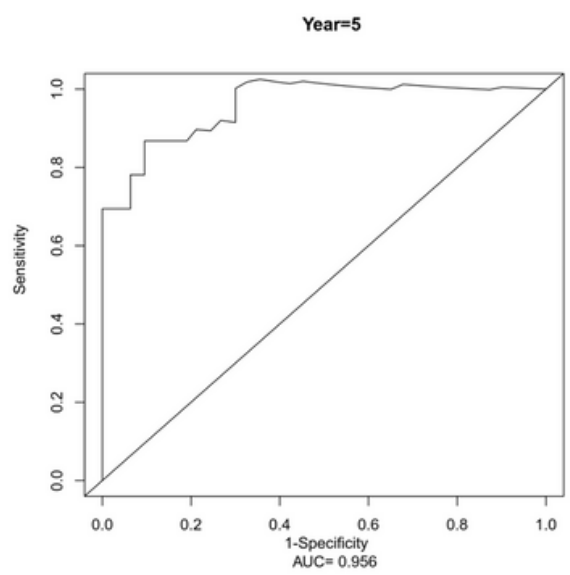

\section{Figure 4}

A. Calibration plot for the nomogram in the test cohort. B. The AUC of 1-, 3-, 5-year DFS in the test cohort. 